\title{
REPORTE DE CASO
}

\section{Espondilodiscitis por Chryseobacterium indolgenes Spondylodiscitis by Chryseobacterium indolgenes}

\author{
Carmen Teresa Esquivia Pájaro, María Fernanda Vásquez Rojas
}

\section{RESUMEN}

La espondilodiscitis es una infección del cuerpo vertebral y del disco intervertebral, cuyas manifestaciones clínicas iniciales pueden confundirse con el dolor lumbar mecánico.

Es importante un diagnóstico rápido, y tener en cuenta los factores de riesgo que pueden favorecer su desarrollo. La gestión temprana puede prevenir la discapacidad causada por esta enfermedad.

La incidencia de esta entidad en la sociedad occidental varía de 0,4 a 2,4 por 100.000 habitantes al año y diferentes comorbilidades aumentan el riesgo: diabetes, uso de drogas intravenosas, alcoholismo, cirrosis hepática, cáncer, insuficiencia renal,

Autores:

Carmen Teresa Esquivia Pájaro Especialista en Medicina Física y Rehabilitación. Jefe del Servicio de Medicina Física y Rehabilitación, Hospital Militar Central, Bogotá D. C., Colombia.

María Fernanda Vásquez Rojas Residente II año de Medicina Física y Rehabilitación. Universidad Militar Nueva Granada, Hospital Militar Central, Bogotá D. C., Colombia.

Correspondencia: carmente6@yahoo.es

Recibido:

15 octubre de 2016

Aceptado:

17 noviembre de 2017

Conflictos de interés:

Ninguno inmunosupresión, procedimientos espinales invasivos o infecciones que afectan el sistema genitourinario y gastrointestinal.

Palabras clave: Espondilodiscitis, dolor lumbar mecánico, Chryseobacterium indolgenes.

\section{ABSTRACT}

Spondylodiscitis is an infection of the vertebral body and the intervertebral disc whose initial clinical manifestations can be confused with mechanical low back pain.

It is important a rapid diagnosis, and to take into account risk factors that can favor its development. Early management can prevent the disability caused by this disease.

The incidence of this entity in Western society varies from 0.4 to 2.4 per 100,000 inhabitants per year, and different comorbidities increase the risk: diabetes, intravenous drug use, alcoholism, liver cirrhosis, cancer, renal failure, immunosuppression, invasive spinal procedures or infections affecting the genitourinary and gastrointestinal system.

Key words: Spondylodiscitis, low back pain, Chryseobacterium indolgenes 


\section{INTRODUCCIÓN}

La espondilodiscitis es una infección del cuerpo vertebral y el disco intervertebral cuya clínica en fases iniciales se puede confundir con cuadros de dolor lumbar mecánico. Es necesario un elevado grado de sospecha clínica para evitar y retrasar su diagnóstico, así como tener en cuenta la presencia de factores de riesgo que pudieran favorecer su desarrollo. La detección y el manejo precoz pueden evitar en gran parte la discapacidad que causa este cuadro.

La incidencia de esta entidad en la sociedad occidental varía de 0,4 a 2,4 por cada 100.000 habitantes al año, distribuyéndose en dos picos de edad, el primero en la primera infancia y el segundo alrededor de la sexta década. Se ha encontrado que un número de comorbilidades influye para aumentar el riesgo, entre ellas la diabetes, el uso de drogas intravenosas, alcoholismo, cirrosis hepática, cáncer, insuficiencia renal, inmunosupresión, procedimientos invasivos en la columna o las infecciones que afectan el sistema genitourinario y gastrointestinal. La mayoría de los casos se deben a diseminación hematógena de infecciones a distancia, siendo menos frecuentes las causadas por propagación de infecciones desde tejidos adyacentes y las debidas a procesos invasivos de la columna.

El objetivo del presente artículo es mostrar, a propósito, el reporte de un caso de espondilodiscitis por un microorganismo inusual en el periodo 2014-2015.

\section{REPORTE DE CASO}

Paciente masculino de 77 años con antecedente de esplenectomía de larga data por accidente de tránsito. Presentó cuadro de 3 meses de dolor lumbar de características mecánicas, dolor a la palpación lumbosacra, sin déficit sensitivo o motor, adecuados arcos de movilidad. Con persistencia de cuadro doloroso y mala respuesta al manejo analgésico y de terapia física, se solicitó radiografía lumbar reportada con múltiples osteofitos, disminución de espacio articular L3-L4 y fusión del cuerpo vertebral L1-L2 con posible fractura patológica sobre L1. Figura 1.

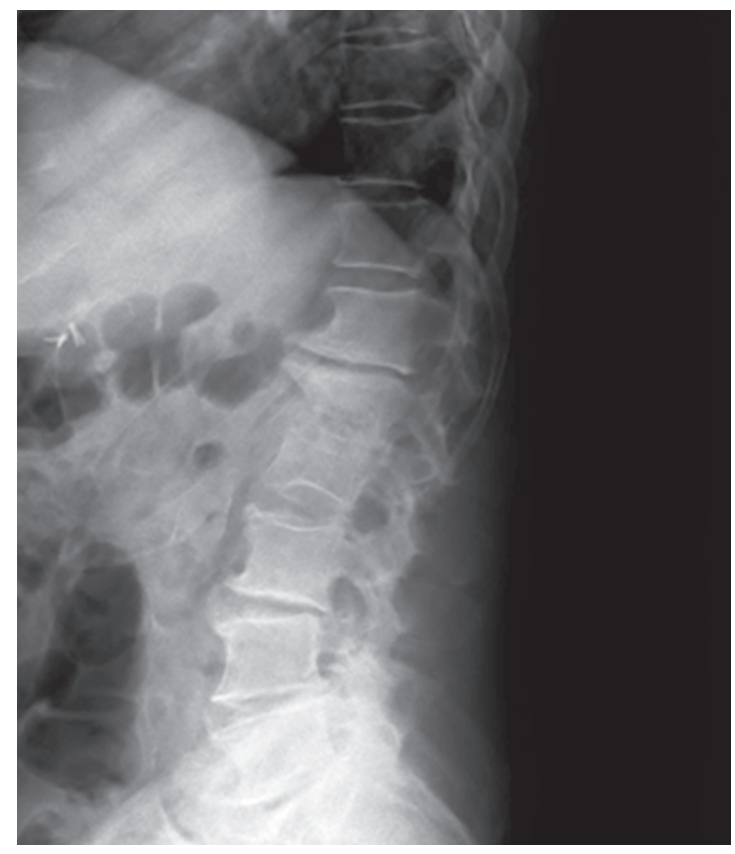

Figura 1. Radiografía lateral de columna lumbar. Fusión del cuerpo vertebral L1-L2, pérdida del disco intervertebral.

Consultó en varias oportunidades por persistencia de dolor sin presentar signos de compresión medular ni déficit neurológico, por lo que lo manejaban de forma sintomática y ambulatorio. Pero dada la persistencia de dos meses del cuadro doloroso, que empeoraba con la movilización, limitaba la marcha, asociado a retención urinaria e hipoestesia en silla de montar, se solicita resonancia magnética de columna lumbar reportada con cambios inflamatorios perivertebrales y epidurales anteriores con disminución de la amplitud de recesos laterales y agujeros de conjunción sobre L1-L2, y gamagrafía ósea con lesiones hipercaptantes.

Dados estos hallazgos imagenológicos y la persistencia de sintomatología, se hospitaliza con impresión diagnóstica de espondilodiscitis versus infiltración tumoral. Se realiza biopsia de cuerpo vertebral L2 con reporte de inflama- 
ción aguda y crónica negativa de malignidad y cultivo de hueso que evidencia bacilos gram negativos, y microorganismo Chryseobacterium indolgenes resistente a carbapenems, y paraclínicos complementarios sin signos de respuesta inflamatoria sistémica ni repercusión hemodinámica. Al examen físico se halla con signos de banderas rojas dados por disminución en fuerza de miembros inferiores, hipoestesia en silla de montar e incontinencia urinaria. Durante su estancia hospitalaria requiere uso de opioides potentes, además de antibiótico por 2 semanas antes de realizar procedimiento quirúrgico para artrodesis posterior, colocación de ganchos laminares pediculares desde cuerpo vertebral T11 hasta cuerpo vertebral L4 y colocación de cilindro somático. Figura 2.

En el posoperatorio se inicia terapia física de forma temprana para evitar el desacondicionamiento físico, terapia sedativa para modular vías del dolor, y terapia ocupacional enfocada en reentrenamiento de transferencia segura, marcha y deambulación.

El paciente tuvo una estancia hospitalaria de 30 días completando manejo antibiótico, disminución progresiva del opioide y manejo rehabilitador, con mejoría sintomática, restauración de la marcha y sin complicaciones seculares.

\section{DISCUSIÓN}

Se presenta el caso clínico de un paciente con dolor lumbar de características mecánicas con mala respuesta al manejo médico convencional y empeoramiento sintomático; con factores de riesgo para desarrollar espondilodiscitis como la edad, inmunosupresión secundaria por esplenectomía, cambios imagenológicos y reporte de patología en cultivo de hueso positivo para bacteria atípica y no reportado en la literatura, Chryseobacterium indologen, un microrganismo gram negativo, anaerobio facultativo, que se puede encontrar en el suelo, las plantas, los alimentos, las fuentes de agua potable cloradas y los insumos médicos. Dicho microorganismo se asocia a infecciones en

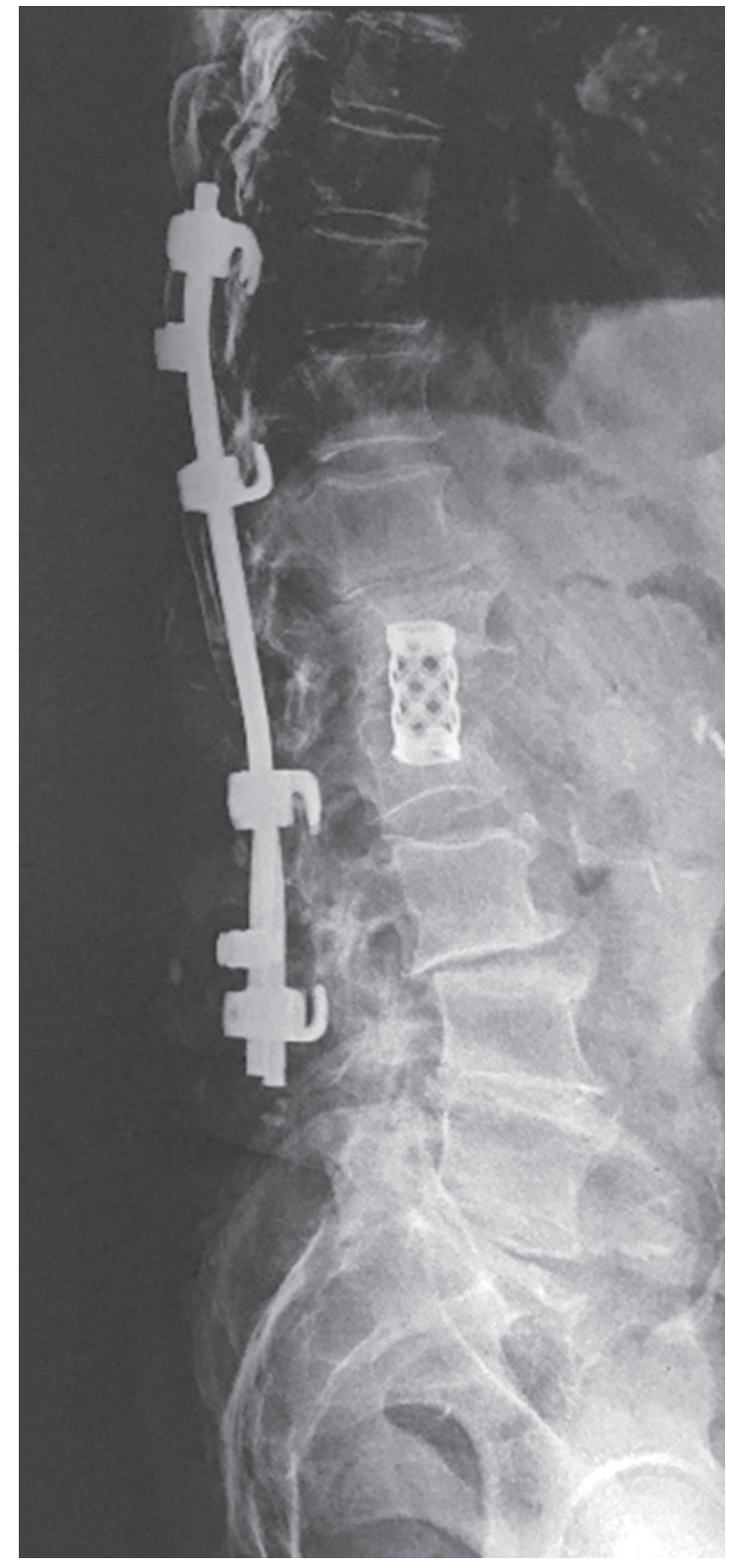

Figura 2. Radiografía lateral de columna lumbar. Artrodesis posterior T11-L4 con cilindro expansor.

población inmunocomprometida, procedimientos invasivos como uso de catéteres, infecciones del tracto urinario o gastrointestinal y diabetes.

En correlación con la casuística mundial, la incidencia más alta de los microorganismos causales proviene de las bacterias gram positivas como Staphylococcus aureus (57\%), estreptococos $(2,0 \%)$, enterococos $(0,7 \%)$ y bacterias anaerobias (menos del 1\%). 
No hay reportes de la asociación de Chryseobacterium indologen con presentaciones de espondilodiscitis.

\section{CONCLUSIÓN}

Esta entidad requiere un grado de suspicacia elevado en pacientes con dolor lumbar sin mejora al manejo convencional. Se hace hincapié en la sospecha inicial, la localización a través de imágenes y la identificación de banderas rojas como la detección de comorbilidades, ya que son el punto de partida y hacen parte de un tratamiento precoz para evitar situaciones de discapacidad en el futuro.

\section{CONFLICTO DE INTERESES}

Los autores manifiestan no tener conflicto de intereses en este estudio.

\section{RESPONSABILIDADES ÉTICAS}

\section{Protección de personas y animales}

Los autores declaran que para esta investigación no se han realizado experimentos en seres humanos ni en animales.

\section{CONFIDENCIALIDAD DE LOS DATOS}

Los autores declaran que en este artículo no aparecen datos de pacientes.
Derecho a la privacidad y consentimiento informado.

Los autores declaran que en este artículo no aparecen datos de pacientes.

\section{REFERENCIAS}

1. Cottle L, Riordan T. Infectious spondylodiscitis. J Infect. Junio de 2008; 56(6): 401-412.

2. Skaf GS, Kanafani ZA, Araj GF, Kanj SS. Non-pyogenic infections of the spine. Int $J$ Antimicrob Agents. Agosto de 2010; 36(2): 99-105.

3. Skaf GS, Domloj NT, Fehlings MG, Bouclaous CH, Sabbagh AS, Kanafani ZA, et al. Pyogenic spondylodiscitis: an overview. J Infect Public Health. 2010; 3(1): 5-16.

4. Rutges JPHJ, Kempen DH, van Dijk M, Oner FC. Outcome of conservative and surgical treatment of pyogenic spondylodiscitis: a systematic literature review. Eur Spine J Off Publ Eur Spine Soc Eur Spinal Deform Soc Eur Sect Cerv Spine Res Soc. Abril de 2016; 25(4): 983-999.

5. Gouliouris T, Aliyu SH, Brown NM. Spondylodiscitis: update on diagnosis and management. J Antimicrob Chemother. Noviembre de 2010; 65 Suppl 3: iii11-24. 
